\title{
Passenger Wait Time Perceptions at Bus Stops: Empirical Results and Impact on Evaluating Real- Time Bus Arrival Information
}

\author{
Rabi G. Mishalani and Mark M. McCord, Ohio State University \\ John Wirtz, Edwards and Kelcey, Inc., Chicago, Illinois
}

\begin{abstract}
This study quantifies the relationship between perceived and actual waiting times experienced by passengers awaiting the arrival of $a$ bus at a bus stop. Understanding such a relationship would be useful in quantifying the value of providing realtime information to passengers on the time until the next bus is expected to arrive at a bus stop. Data on perceived and actual passenger waiting times, along with socioeconomic characteristics, were collected at bus stops where no real-time bus arrival information is provided, and relationships between perceived and actual waiting times are estimated. The results indicate that passengers do perceive time to be greater than the actual amount of time waited. However, the hypothesis that the rate of change of perceived time does not vary with respect to the actual waiting time could not be rejected (over a range of 3 to 15 minutes). Assuming that a passenger's perceived waiting time is equal to the actual time when presented with accurate real-time bus arrival information, the value of the eliminated additional time is assessed in the form of reduced vehicle hours per day resulting from a longer headway that produces the same mean passenger waiting time. The eliminated additional time is also assessed in the form of uncertainty in the headway resulting in the same extra waiting time. Naturally, such benefits of passenger information can
\end{abstract}


only be confirmed when the actual effect of information on the perception of waiting time is quantified.

\section{Motivation and Hypothesis}

The background motivating this study is first discussed, and the objective is then presented. Real-time bus arrival information-for example, delivered to prospective passengers waiting at bus stops via variable messages signs (VMSs) - can be useful to transit passengers for a multitude of reasons. Passengers can use their waiting time more productively, select which route they would want to take, or choose to select an alternative mode of transportation. Whatever the prospective passengers' choices are, providing them with real-time information reduces the uncertainty inherent to transit systems. Empirical evidence shows that the time travelers spend outside the transportation vehicle of choice (e.g., waiting at a stop) is more onerous than the time they spend inside the vehicle in motion to their destination (Ben-Akiva and Lerman 1985). This is partly due to the higher degree of uncertainty associated with waiting for a transit vehicle. This phenomenon is well characterized by Duffy (2002): "People don't mind waiting for a bus if they know how long it's going to be. Even if they have to waste the time, at least they know it's going to be 15 minutes. Otherwise they're sitting there thinking the bus will be along in about two minutes, and when it doesn't show, then they start getting frustrated." In general, reducing waiting time uncertainty is expected to improve passenger satisfaction, and ultimately increase bus ridership.

Mishalani et al. (2000) studied the value of information to passengers in terms of using the waiting time more effectively, while Hickman and Wilson (1995) studied the value in terms of improved route choice. This research focuses on passengers' perceptions of their waiting time at stops (outside the vehicle) and, as a result, the possible reduction in such times when real-time passenger information is provided. To study the perceptions of waiting time, a survey of prospective passengers at bus stops was conducted. The collected data were then analyzed whereby the relationship between perceived and actual waiting times was investigated.

The main hypothesis of this study is that without real-time bus arrival information, passengers are likely to perceive waiting time to be greater than it actually is. When accurate bus arrival information is provided, it is assumed that passengers will perceive their waiting time to be equal to the actual waiting time. In this case, a passenger will arrive at a stop and look at the VMS, which will display the min- 
utes until the next bus is expected to arrive on the route of interest. The VMS will continue to update the expected time while the passenger waits.

Without bus arrival information provided to passengers, the relationship between perceived and actual waiting times is expected to follow a function where the perceived time is greater than the actual time. The form of the function might depend on the magnitude of the waiting time. For example, the additional time due to exaggerated perceptions may be further magnified under long waits in comparison to short waits. One can also imagine the opposite situation, where longer waits may be perceived more accurately due to the more conscious recognition of time under such conditions.

The main objective of this study is to model and quantify the difference between perceived and actual passenger waiting times at bus stops in the absence of accurate real-time bus arrival information and to investigate the effects of duration of the actual waiting time and socioeconomic variables on this difference. This objective is achieved in the context of a pilot study by estimating models that describe waiting time as perceived by passengers waiting for buses at stops. To do so, appropriate field data were collected. Once the difference between perceived and actual waiting times were quantified, possible factors that might affect the magnitude of the difference were explored. Moreover, an analysis of the potential benefits of providing accurate bus arrival time information at bus stops was carried out.

\section{Data Collection}

Data were collected by surveying passengers waiting at bus stops for Campus Area Bus Service (CABS) buses, which are operated by the Transportation and Parking Office of Ohio State University in Columbus. CABS serves the campus community, which includes close to 50,000 students, resulting in an annual ridership of approximately 4 million. The operation consists of 15 to 2040 -foot buses running simultaneously on several routes of lengths ranging from $2 \mathrm{~km}$ to $8 \mathrm{~km}$ on and in the areas surrounding the campus.

The transit service used in this pilot study is small enough to be manageable, yet large enough to reflect situations pertinent to more extensive transit services in urban areas. Nevertheless, it would be important to build upon this pilot study in future research by examining larger transit systems. Extensions to larger systems would render the findings more applicable to a wider set of conditions, most notably, longer routes and more heterogeneous traveling populations. 
Three students surveyed 83 passengers over a period of approximately one year, from spring 2001 to spring 2002. A surveyor went to a bus stop, noted the arrival time of a passenger, and later asked him or her a series of questions. A response rate close to 100 percent was achieved.

\section{Bus Stops}

A set of appropriate bus stops was first determined for the purpose of conducting the surveys on the basis of four criteria. The first criterion was to choose a stop that does not serve many routes. In fact, a stop that serves only one bus route is ideal. Fewer routes will help the interviewer know, or possibly guess, which route a passenger is going to choose before he or she gets on the bus. A surveyor sitting at a stop has a general idea of when the next bus will arrive based on both the published headway and observations of the buses over a period of time. If a bus stop serves many different routes, it becomes more difficult, if not impossible, to know the route a random passenger plans to use. The passenger might then board a bus before the surveyor has a chance to conduct the interview, thus missing a data collection opportunity.

The second criterion for selecting a good bus stop for surveying purposes is to use a stop that serves routes with longer headways. Longer headways are attractive because it is desirable to have the option to survey a passenger after a notable wait. While data with short waiting times are needed for a complete data set, not all the data should be collected after a 3- to 4-minute wait. A longer headway allows the interviewer more options on when to survey passengers and observe longer waits.

One issue with longer headways, though, is whether a schedule is published. This issue leads to the third criterion. It is helpful to select bus stops where passengers arrive totally randomly. Such arrivals typically occur when only the headway on a route is published, rather than the scheduled time of bus arrivals. When schedules are published and headways are long, most passengers will likely arrive shortly before published arrival times, thus reducing the opportunity of observing relatively longer waiting times.

Finally, it is productive to collect data at stops with relatively high demand. Passengers must arrive frequently at a bus stop to ensure that interviewers do not experience a large amount of idle time. Otherwise, longer survey times will be needed to produce the same number of observations. Based on the above criteria, a total of five stops on three routes were selected. Two of the routes have published head- 
ways of 10 minutes and one a published headway of 15 minutes. None, however, has a published schedule.

\section{Interviews and Observations}

During the survey process, when a passenger arrived at the bus stop, the arrival time was noted. The first one or two passengers that arrived after the previous bus departure were selected. This selection strategy ensured that passengers with a wider range of wait times were interviewed and that the interviewer was able to survey them without the bus coming before the interview was complete. The interviewer decided when to survey the passenger. This is selected largely on when the next bus was thought to be arriving. The interviewer typically began the interview with a passenger at least a minute before the expected bus arrival to ensure enough time to complete the interview without the passenger feeling anxious about catching the bus. As already discussed, the interviewers had a good idea as to when the next bus would arrive because of their knowledge of the previous bus arrival time and service operations.

In addition to the surveyor's name, date, and weather conditions, the following is a list of variables observed for each interviewed waiting passenger:

1. origin of the passenger (bus stop where the survey is conducted);

2. passenger's arrival time to the stop;

3. time the passenger was surveyed;

4. passenger's gender;

5. passenger's race;

6. passenger's perceived waiting time;

7. whether the passenger was wearing a watch;

8. destination of the passenger;

9. maximum time the passenger would be willing to wait were real-time bus arrival information provided;

10. approximate walking time to the destination from the bus stop at which the passenger was waiting;

11. whether the passenger had a time constraint (such as making it to a class at a certain time);

12. familiarity of the passenger with the transit service, as measured by frequency of use in number of trips per day; 
13. passenger's car ownership status; and

14. passenger's affiliation with the university (undergraduate student, graduate student, staff, faculty, or visitor).

Items 1 through 7 were always recorded by the surveyor, while items 8 through 14 were generally collected, time permitting. The first question, asked after a brief introduction, was "In minutes, how long do you think you have been waiting?" It was important to specify the desired level of accuracy to the passengers to avoid the possibility of their rounding off to the nearest 5 minutes.

\section{Perceived Waiting Time Models}

The model development and estimation aimed at quantifying the difference between perceived and actual waiting times, along with the exploration of factors influencing that difference, are discussed in this section. The modeling consists of two parts. First, a simple ordinary least squares (OLS) regression model of perceived versus actual waiting times is estimated. Hypothesis tests are then applied to determine whether a significant difference between the two variables exists and to assess the nature of the relationship. Second, the impact of socioeconomic variables on the relationship between perceived and actual waiting times is investigated.

\section{Perceived versus Actual Waiting Times}

A simple linear regression model of the following form is estimated:

$p=\beta_{0}+\beta_{1} a+\varepsilon$

where:

$\mathrm{p} \quad$ is the perceived waiting time

a represents the actual waiting time

$\beta_{0} \quad$ is the parameter representing the intercept of the regression line

$\beta_{1} \quad$ denotes the parameter representing the slope of the regression line

$\varepsilon \quad$ is a random variable with mean of 0 
The estimation results, shown in Table 1, indicate that the perceived waiting time is greater than the actual within the range of the data set whereby the actual waiting time varies between 3 and 15 minutes. However, it is important to apply statistical hypothesis testing to assess the significance of this finding.

\section{Table 1. Estimation Results of Perceived vs. Actual Waiting Times}

\begin{tabular}{lccr}
\hline Variable & Est. Parameter & Standard Error & t-statistic \\
\hline Intercept & 1.33 & 0.48 & 2.77 \\
Actual wait time & 0.92 & 0.076 & 11.96 \\
\hline No. of observations $=83, \overline{\mathrm{R}}^{2}=0.634$ & & \\
\hline
\end{tabular}

In addition to the fairly high corrected goodness-of-fit measure $\overline{\mathrm{R}}^{2}$, the parameter estimate $\hat{\beta}_{1}$ with a t-statistic of 11.96 is significantly different from 0 at the 0.01 level, clearly indicating that the perceived waiting time varies positively with the actual waiting time. More interestingly, though, is the null hypothesis that the parameter estimate $\hat{\beta}_{1}$ is equal to 1 . For the general case when testing whether a parameter estimate is equal to some non- 0 value, the $t$ statistic is given by Pindyck and Rubinfeld (1998). In the case of the null hypothesis of interest, namely that $\hat{\beta}_{1}=1$, the $t$-statistic takes the value of -1.11 (see Wirtz [2002] for the details behind this calculation), resulting in the failure to reject the hypothesis at the 0.10 level. That is, the slope of the regression line is not statistically different from 1 and, hence, the data do not support the notion that passengers perceive time any more or less differently after waiting for longer periods.

In addition to the actual time ranging between 3 and 15 minutes in the data set, 94 percent of the actual waiting time observations range between 3 and 9 minutes. Therefore, it is quite possible that, under longer waiting times, the perceptions may increase at a different rate than unity with respect to the actual time. Moreover, given that the rejection of a hypothesis does not imply its acceptance, it is also worth pointing out that the less-than- 1 value of the estimated slope means that (on the basis of the parameter estimates of Table 1), as the wait gets longer, the exaggerated perceptions become more closely aligned with reality for actual waiting times as long as 15 minutes. This observation is consistent with the notion that longer waits are associated with more accurate perceptions of time. 
In any event, the failure to reject the hypothesis that $\hat{\beta}_{1}$ is equal to 1 renders the testing of the additional null hypothesis that $\hat{\beta}_{0}$ is equal to 0 more critical. More specifically, only a parameter estimate $\hat{\beta}_{0}$ that is statistically greater than 0 would confirm that the perceived waiting time is greater than the actual waiting time. Indeed, with a t-statistic of 2.77 , the estimate of 1.33 is significantly different from 0 at the 0.01 level.

Thus far, the null hypotheses $\hat{\beta}_{0}=0$ and $\hat{\beta}_{1}=1$ were tested separately. It is also useful to test the two hypotheses jointly using an F-test (Pindyck and Rubinfeld 1998) where the joint null hypothesis is $\hat{\beta}_{0}=0$ and $\hat{\beta}_{1}=1$, which reflects the function $p=a+\varepsilon$. In other words, on average, the perceived waiting time equals the actual waiting time. If this test fails to reject the null hypothesis, there is no statistical difference between what passengers perceive and the actual waiting time. The F-statistic for the hypothesis of interest is 10.46 (see Wirtz [2002] for the details behind the calculation). This value implies the rejection of the null hypothesis at the 0.01 level (the critical value corresponding to this level is 4.91), thus confirming that passengers do perceive waiting times to be greater than actual times, at least in the range between 3 and 15 minutes.

Finally, it is worth pointing out that the finding that perceived waiting times are statistically greater than actual waiting times within the range of the data identifies one possible contributor to the well-established conclusion that out of vehicle travel time (of which waiting time at a bus stop is a possible component) is more onerous to travelers than in-vehicle travel time (Ben Akiva and Lerman 1985).

\section{Socioeconomic Variables}

Introducing socioeconomic variables to the original specification of equation (1) is also considered. Investigating the socioeconomic variables listed above, none are found to have parameter estimates significantly different from 0 at the 0.05 level. This result could partly be attributed to the fact that data on some of these variables were not collected for each interviewed passenger because of time constraints during the survey process. For some variables, the number of observations is as low as 46. Nevertheless, it is worthwhile to point out two variables that exhibit some noteworthy effect.

In particular, the walking time to the destination from the bus stop at which the passenger is waiting and the presence of a time constraint do exhibit some explanatory value. Data on both variables were provided by passengers in response to interview questions. The former is measured in minutes, and the latter is a dummy 
variable that takes a value of 1 if a time constraint is present and 0 otherwise. These two variables are included in the model as follows:

$p=\beta_{0}+\beta_{1} a+\beta_{2} w+\beta_{3} c+\varepsilon$

where:

$w \quad$ is the walking time from the bus stop the passenger is waiting at to the destination

c represents the time constraint dummy variable

$\beta_{2}$ and $\beta_{3}$ are parameters

The details behind the identification of these variables are discussed in Wirtz (2002). The estimation results are given in Table 2. The $t$-statistics of the estimates of the intercept and the parameters of the two additional variables reflect values significantly different from 0 at the 0.10 level. Furthermore, the null hypothesis that $\hat{\beta}_{2}=\hat{\beta}_{3}=0$ is rejected at the 0.10 level by applying the $\mathrm{F}$-test, indicating that this model adds significant explanatory value (at the 0.10 level) with respect to the original model.

\section{Table 2. Estimation Results with Socioeconomic Variables Included}

\begin{tabular}{lccr}
\hline Variable & Est. Parameter & Standard Error & t-statistic \\
\hline Intercept & 1.68 & 0.94 & 1.80 \\
Actual wait time & 0.77 & 0.18 & 4.36 \\
Walking time & 0.047 & 0.028 & 1.71 \\
Time constraint & -0.91 & 0.62 & -1.47 \\
\hline No. of observations $=46, \overline{\mathrm{R}}^{2}=0.529$ & & \\
\hline
\end{tabular}

One possible explanation of the positive parameter value of the walking time to destination variable is that a passenger going farther is more dependent on the bus service (i.e., practically, the passenger does not have an alternative transportation option for the desired trip on the university campus) and is therefore likely to be 
more frustrated from repeatedly having to take the bus than a passenger taking the bus only occasionally. Such frustration would result in a further exaggeration of perceived waiting time. Moreover, passengers with shorter walk times who choose to take the bus rather than walk to their destination are likely to be less sensitive to the time of their wait-given the choice that they have made-than those with longer walking times.

As for the presence of the time constraint variable, one possible explanation for the negative sign of its corresponding parameter estimate is that a passenger with a time constraint is more conscious of how much time is actually elapsing. Being conscious of the elapsed time would tend to lessen exaggerations in misperceptions compared to the case when a time constraint is not present. Alternatively, one might expect the opposite effect, whereby the presence of a time constraint introduces anxiety that leads to an increase in the perceived waiting time. The negative value of the parameter estimate corresponding to the time constraint dummy variable supports the dominance of the former effect. Future studies employing more comprehensive date sets would shed more light on this interesting finding. Finally, similar hypothesis tests to those applied to the original model (i.e., Table 1) are also applied to the model including the walking time and time constraint variables (i.e., Table 2). As in the case of the original model, the t-test fails to reject the hypothesis that the parameter associated with the actual waiting time is equal to 1 at the 0.05 level. Moreover, the estimate of the intercept is significantly different from 0 at approximately the 0.07 level.

\section{Potential Value of Providing Passenger Information}

Empirically, the mean perceived waiting time of the passengers surveyed is 6.61 minutes, while their mean actual waiting time is 5.77 minutes. In light of the quantified difference of 0.84 minutes, the assessment of the value of providing passenger information on the expected time until the next bus arrival consists of two parts. First, the additional 0.84 minutes of perceived waiting time are converted to equivalent vehicle-hours per day saved if real-time information is provided at stops while maintaining the same mean perceived passenger waiting time, under the assumption of deterministic headways. Second, the additional 0.84 minutes of perceived waiting time are converted into an equivalent headway uncertainty. In the event that the effects of actual waiting time and socioeconomic variables on the difference between the perceived and actual waiting time are found to be statistically significant in the context of a more extensive study, the expected dif- 
ference given the conditions of interest-instead of the unconditional difference of 0.84 minutes-would be used in the forthcoming analyses.

In both cases, it is assumed that real-time passenger information will eliminate exaggerated perceptions of waiting time. Thus, the two measures provide an assessment of the value of introducing such information in terms of known performance measures. As illustrated below, the introduction of real-time information systems could result in a reduced operating cost or an increased passenger satisfaction. Such benefits could ultimately lead to increased ridership for public transit, depending on the policies adopted by the transit agency in conjunction with the introduction of such information systems.

Naturally, the subsequent discussion of the value of introducing real-time passenger information depends on the validity of the assumption that such information will produce accurate perceived waiting times. While this assumption requires further research to be validated, it is not unreasonable to adopt this assumption in the absence of evidence suggesting otherwise.

\section{Equivalent Vehicle-Hours}

If the exaggeration in the perceptions of waiting time is eliminated by providing real-time passenger information, the quantified difference of 0.84 minutes will be reduced to 0 . Under such a condition, the transit agency can achieve the same level of service, as measured by the mean passenger waiting time, by employing fewer vehicle-hours per day. Decreasing vehicle-hours would translate to reduced labor, energy, and vehicle maintenance costs. Assuming that the headway is deterministic (i.e., the headway variance is 0 ) and passengers arrive in a totally random manner (i.e., arrivals follow the Poisson process), the mean actual passenger waiting time is equal to half the deterministic headway. Furthermore, under deterministic headways and running times, the vehicle-hours per day required for service provision is the ratio of the running time to the headway multiplied by the total hours of service provided per day. Naturally, for a given running time, fewer vehicle-hours per day are required when the headway is longer.

In the event that real-time information is provided and the additional mean of 0.84 minutes of waiting time as perceived by passengers is eliminated, operating at a headway of $h+2 \times 0.84$ minutes-where $h$ is the headway when real-time passenger information is not provided-will result in the same mean perceived passenger waiting time as that when information is not provided and waiting times are exag- 
gerated due to passenger perceptions. As a result, the percent reduction $Z$ in the vehicle hours per day required for service provision is given by the following:

$Z=1.68(h+1.68)^{-1}$

This function is plotted in Figure 1 for values of mean headway ranging from 5 to 30 minutes where the percent reduction in vehicles-hours per day varies between 25.1 and 5.3 percent.

A typical CABS route has a mean running time of 30 minutes, a mean headway of 10 minutes, and operates over a period of 18 hours during a weekday. Under these operating conditions, providing real-time information while maintaining the same mean passenger waiting time results in a reduction of 7.77 vehicle-hours per day from the original total of 54 , amounting to a 14.4 percent saving (as seen in Figure 1).

Figure 1. Percent Reduction in Vehicle-Hours per Day vs. Headway

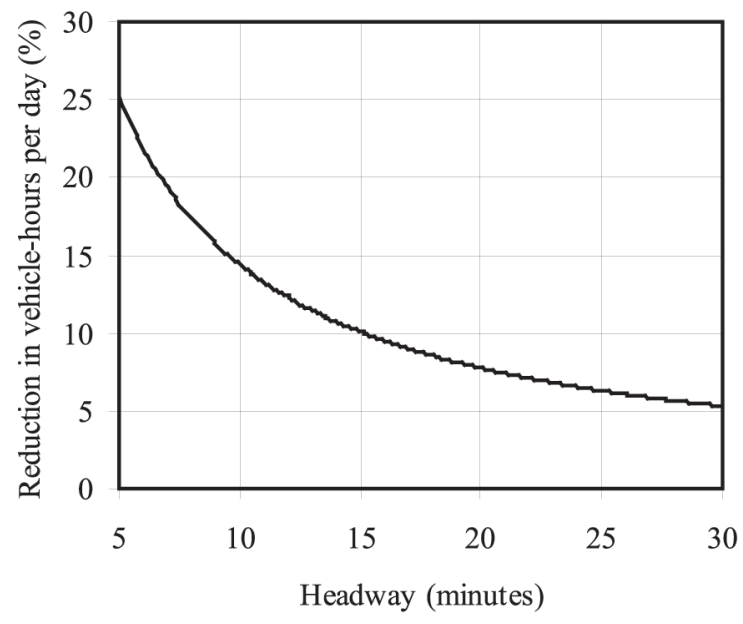




\section{Equivalent Coefficient of Variation}

In this exercise, the difference of 0.84 minutes is converted to an equivalent coefficient of variation in the headway of a route that would increase mean passenger waiting times by 0.84 minutes with respect to the mean wait time under a deterministic headway. In this case, the value of real-time information is assessed in terms of eliminating a certain level of uncertainty in the headway. Assuming that passengers arrive in a totally random manner, the expected waiting time is given by the following (Larson and Odoni 1981):

$$
\mathrm{E}[w]=\frac{\mathrm{E}[h]}{2} \cdot\left(1+\frac{\operatorname{Var}[h]}{\mathrm{E}[h]^{2}}\right)=\frac{\mathrm{E}[h]}{2} \cdot\left(1+\mathrm{CV}[h]^{2}\right)
$$

where:

$E[w] \quad$ is the expected waiting time for a randomly arriving passenger

$E[h] \quad$ represents the expected headway

$\operatorname{Var}[h]$ denotes the variance of the headway

$\mathrm{CV}[\mathrm{h}]$ is the coefficient of variation of headway (the ratio of the stan dard deviation to the mean)

To obtain the coefficient of variation of headway that results in an increase in mean waiting time equivalent to the difference between the perceived and actual waiting times, $\mathrm{CV}[h]$ is solved for using equation (4) for given values of $\mathrm{E}[w]$ and $E[h]$, where the difference $E[w]-E[h] / 2$ remains equal to 0.84 minutes. (Note: $E[h] / 2$ is the mean waiting time when the headway is deterministic.) Naturally, the result depends on the value of $E[h]$, and is given by the following:

$\mathrm{CV}[h]=1.296 \mathrm{E}[h]^{-1 / 2}$

This function is plotted in Figure 2 for values of mean headway ranging from 5 to 30 minutes. The equivalent coefficient of variation is seen to vary between 0.580 and 0.237 . 
The equivalent coefficient of variation of headway is a measure of the bus service quality as perceived by passengers. Elimination of increased perceived waiting times on a bus route that does not exhibit any variability in its headway could conceptually be traded off against increased variability in headways. For example, an increased passenger wait time perception of 0.84 minutes on a route with a deterministic headway of 10 minutes has an equivalent headway coefficient of variation of 0.410 , as seen in Figure 2, on a route with random headways and wait time perceptions equal to actual wait times. This value of $\mathrm{CV}[h]$ corresponds to a headway standard deviation of 4.10 minutes. As an illustration, such a standard deviation could be produced by a total of two buses bunching, with a shorter headway of 7.1 minutes and a longer headway of 12.9 minutes. Thus, for the data set used in this study and a 10-minute headway, eliminating exaggerated perceived waiting times by providing accurate real-time information on the time until the next bus is expected to arrive can be thought of as equivalent to eliminating bus bunching producing a headway standard deviation of 4.10 minutes.

\section{Figure 2. Equivalent Coefficient of Variation vs. Expected Headway}

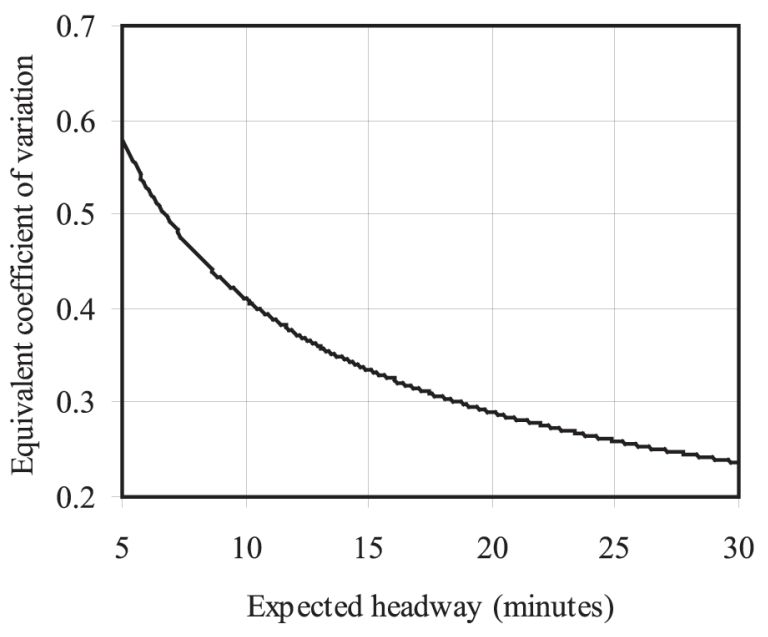

\section{Summary and Future Research}

Through the observation of 83 prospective passengers waiting at bus stops, the relationship between perceived and actual waiting times was investigated. The 
results of estimating a linear relationship between the two variables indicate that, while the intercept is significantly greater than 0 , the slope's equality to 1 cannot be rejected. This finding implies that the data do not reveal evidence that the additional perceived waiting time varies with the actual time within the range of 3 to 15 minutes reflected in the data set. Moreover, some socioeconomic variables are found to have explanatory value. In particular, a passenger's walking time to the destination from the bus stop at which he or she is waiting and the presence of a time constraint reflect an impact on the perceived waiting time. A longer walking time produces a greater exaggeration in the perceived waiting time, while the presence of a time constraint brings the perceived waiting time closer to the actual time.

The mean difference between the perceived waiting time by passengers and the actual time is 0.84 minutes. This additional time is related to both an equivalent savings in vehicle-hours per day and an equivalent coefficient of variation (the ratio of the standard deviation to the mean) in the headway of a route. These relationships are developed in an effort to assess the benefits of eliminating the exaggeration in passengers' perceptions of waiting time by providing accurate realtime passenger information on the time until the next bus arrival. If the additional amount of time-eliminated by the provision of real-time information-is used to increase the headway to a value such that the mean passenger perceived waiting time remains unchanged, the reduction in the vehicles-hours per day required to provide service is derived assuming a deterministic headway and totally random passenger arrivals. For a running time of 30 minutes, a headway of 10 minutes, and a duration of service of 18 hours per day, a reduction of 7.77 vehicle-hours per day is achieved, amounting to a 14.4 percent saving.

If the additional 0.84 minutes of perceived time is added to the expected waiting time of a passenger waiting for a bus when there is no variation in the headway and assuming totally random passenger arrivals, the equivalent headway coefficient of variation $\mathrm{CV}[h]$ producing such an additional waiting time is derived. For a mean headway of 10 minutes, $C V[h]$ is 0.410 reflecting a standard deviation of 4.10 . Thus, providing real-time information at bus stops-for example, via VMSs-could reduce passengers' mean perceived waiting time by an amount equivalent to that achieved through eliminating the corresponding standard deviation in the headway.

Because passengers perceive waiting times to be greater than actual waiting times at a bus stop, real-time passenger information systems could potentially reduce 
the perceived waiting time for buses when providing accurate information. The reduction in perceived waiting times could potentially be translated into reduced operating costs or increased passenger satisfaction and, ultimately, into increased ridership for public transit, depending on the policies adopted by the transit agency in conjunction with the introduction of real-time passenger information systems.

This study demonstrated the feasibility of examining the questions of interest and points to several directions regarding future research. Based on this pilot study, there is good evidence that a difference between perceived and actual waiting times does exist to motivate a more comprehensive data collection and modeling effort. It would be valuable to observe passengers traveling on larger transit systems than the university campus-based system of this study. Larger systems present additional complexity in service that might affect waiting time perceptions (e.g., while waiting during transfers between routes, and when traveling on longer routes) and reflect a more heterogeneous traveling population. A wider range of actual waiting times is also important to allow for testing various specifications characterizing the relationship between perceived and actual waiting times. Moreover, a larger data set with more complete observations of socioeconomic variables is necessary, given the indication that such variables could have an important impact on the perceptions of waiting time. Also, additional socioeconomic variables might influence the perceptions of waiting time and, hence, would be worth observing. Such variables include time of day, whether the passenger has access to time-telling devices other than a watch (e.g., mobile phone, other portable electronics, or public clocks visible from the bus stop), whether the passenger is traveling with a group, and whether a bus operating on a route that does not provide service to the passenger's desired destination arrives while the passenger is still waiting for a service on another route. In addition, in conducting a larger study on a more extensive transit service, which will inevitably present more degrees of freedom, it is important to pay attention to the design of the sampling strategy and to monitor the response rate. Finally, the assumption that passengers perceive waiting time to be equal to the actual time when accurate real-time information is provided should be investigated. In particular, a study whereby passengers are surveyed both before and after the introduction of real time passenger information systems would be particularly valuable. 


\section{Acknowledgments}

This study was funded by the Transportation and Parking Office at the Ohio State University. The support of Sarah Blouch and Steven Basinger of the same office is appreciated. Valuable comments by the anonymous referees are also gratefully acknowledged.

\section{References}

Ben-Akiva, M., and, S.R. Lerman, 1985. Discrete choice analysis. MIT Press.

Duffy, J. 2002. The new face of San Francisco's Muni. http://www.masstransitmag. com/script/search.asp?SearchSiteURL=/articles/2002/mt_05-02/mt_0502_03.htm.

Hickman, M., and N. Wilson, 1995. Passenger travel time and path choice implications of real time transit information. Transportation Research-Part C 3C (4).

Larson, R.C., and A.R. Odoni, 1982. Urban operations research. Prentice Hall.

Mishalani, R., S. Lee, and M. McCord, 2000. Evaluation of real-time bus arrival information systems. Transportation Research Record 1731.

Pindyck, R.S., and D.L. Rubinfeld, 1998. Econometric models and economic forecasts. McGraw Hill.

Wirtz, J. 2002. Passenger perceptions of waiting time at bus stops. BS thesis, Ohio State University, Department of Civil and Environmental Engineering and Geodetic Science.

\section{About the Authors}

RABI G. MishalANI (mishalani@osu.edu) is an associate professor in the Department of Civil and Environmental Engineering and Geodetic Science at Ohio State University. He received his doctor of philosophy degree in civil and environmental engineering from Massachusetts Institute of Technology in 1993, his master of science degree in transportation systems from MIT in 1989, and his bachelor of engineering degree in civil engineering from American University of Beirut in 1987. Prior to joining the faculty at OSU in 1997 , Dr. Mishalan was a research scientist at MIT's Center for Transportation and Logistics. He won the National Science Foundation CAREER award in 2001. His main areas of expertise include the application 
of probability modeling and statistical methods to the planning and managing of transportation systems. His current research focuses on public transit operations in the presence of vehicle location information, highway operations control and traveler guidance and the role of remotely senses traffic data, and infrastructure maintenance decision making in the presence of automated condition data collection technologies.

MARK R. MCCORD (mccord.2@osu.edu) is a professor in the Department of Civil and Environmental Engineering and Geodetic Science at Ohio State University. He received his doctor of philosophy degree in civil engineering from Massachusetts Institute of Technology in 1983, his master of science degree in industrial engineering from Stanford University in 1978, and his bachelor of science degree in civil engineering from Purdue University in 1977. While a doctoral student at MIT, Dr. McCord was a Chateubriand Fellow at Ecole Centrale des Arts et Manufactures in Paris during the 1981-82 academic year. Shortly after joining the faculty at OSU in 1983, he won the National Science Foundation Presidential Fellowship award. His main areas of expertise include the application of decision analysis and subjective probability theory to the planning and managing of transportation systems. His current research focuses on the use of vehicle location data in public transit operations, the assessment of remote sensing technologies in transportation planning, and regional travel demand measurement and forecasting.

JOHN WIRTZ (wirtz.13@osu.edu) is an engineer at Edwards and Kelcey, Inc. in Chicago. He received his master of science degree in civil and environmental engineering from Northwestern University in 2004 and his bachelor of science degree in civil and environmental engineering from Ohio State University in 2002. During both his undergraduate and graduate studies, his research focused on transportation systems analysis and modeling. 